\title{
Effects of microgravity and cosmic radiations on human T lymphocytes
}

\author{
P. Pippia', G. Galleri', M.A. Meloni', A. Saba', G. Pani', M. Cogoli-Greuter'2,A. Cogoli2 \\ I Dipartimento di Scienze Fisiologiche, Biochimiche e Cellulari, Università di Sassari \\ 2 Zero-g Life Tec GmbH, Zurich
}

KEYWORDS: T lymphocytes, microgravity, cosmic radiations, gene expression

\begin{abstract}
In space living organisms, including cells, are affected by two new environmental conditions: microgravity and cosmic radiations. Several experiments in dedicated space missions and in simulated microgravity have shown that low gravity causes a dramatic depression of the mitogenic in vitro activation of Tymphocytes. The goal of this reserch was to determine in space (on board the International Space Station) the ability of adherent monocytes to migrate, as well as to interact with Tcells. A reduced motility of the $\mathrm{J}-1 \mathrm{II}$ cells and changes in the structures of actin, tubulin and vinculin were observed. Moreover, we demonstrated that LFA-I/ ICAM-I interactions occur in space and are dependent on activation time but show differences in number, arrangement and fluorescence intensity, depending on time and experimental conditions. In order to evaluate the effects of cosmic radiations on the gene expression in human T lymphocytes we exposed these cells to high quote cosmic radiation during two stratospheric balloon trans-mediterranean flights (BIRBA missions). The gene expression was analized by CDNA microarray hybridization technology. Activated $T$ cells react to the ionizing stress by activating genes involved in cell cycle check-point, oxidative stress response, heat shock proteins production or by repressing genes involved in antigen recognition.
\end{abstract}

It is well known that during space flight the constant influence of weightlessness leads to several modifications of many physiological processes, such as oxidative metabolism, growth rate signal transduction and gene expression (Cubano and Lewis, 200I). Several alterations are associated with atrophy in heart, muscle and bone (Wang,
1999). Moreover, past experiments in dedicated space missions (sounding rockets and shuttle spacelab) and in simulated microgravity (clinostat) have shown that low gravity affects important cellular processes in human $T$ lymphocytes. In particular, a dramatic depression of the mitogenic in vitro activation (Cogoli et al., 1984) was observed with altered distribution and down regulation of protein kinase C (Cooper and Pellis, 1998), reduced expression of interleukin- 2 and its receptor IL-2R alfa (Walther et al., 1998), alteration in actin, tubulin and vinculin pattern (Sciola et al., 1999), fourfold enhanced 5lipoxygenase activity, increased cytochrome $c$ release and caspase activation, ultimately leading to apoptosis (Maccarrone et al., 2003). The mechanism of T-cell activation is very complex. We supposed that a reduced interaction between $\mathrm{T}$ lymphocytes and monocytes might be one of the reasons for the observed depression of the $T$ cell in vitro activation. Cell motility and a continuous rearrangement of the cytoskeletal network within the cells are essential for cell-cell contacts. The goal of experiment MIA (Motion and InterAct), flown in Kubik BIO\#I, was to determine in space the ability of adherent monocytes to migrate, as well as to interact with T-cells. In the "Motion" experiment monocytes ( $\mathrm{J}-\mathrm{I} I \mathrm{I}$ cells) were incubated on a colloidal gold coated substrate. Migrating cells removed the colloidal gold, leaving a track recording cell motility. Post flight, the migration tracks and the cytoskeletal structures of F-actin, beta-tubulin and vinculin were analysed. A reduced motility of the $\mathrm{J}-\mathrm{I} / \mathrm{I}$ cells and changes in the structures of actin, tubulin and vinculin were observed. Similar results have been found in modeled low gravity (Meloni et al., 2006). Cell-to-cell interactions, mediated by adhesion molecules with the involvement of membrane receptors, play an important role and are involved in signal transduction. It is well known that several cytoskeletal elements are involved in this process. In the "Interact" experiment we studied the effects of microgravity on the interaction of human $T$ lymphocytes with monocytes, investigating the interaction of the leukocyte integrin LFA-I with its ligand ICAM-I. Jurkat $T$ cells and J-I II monocytes were co-cultured in flight and fixed at two different times ( 3 and $18 \mathrm{~h}$ ) after activation. Postflight, the samples were analysed by immunofluorescence technique for cap formation of adhesion proteins LFA-I and ICAM-I. Our results in space are in good agreement with those obtained 
in the RPM clinostat and suggest that LFA-I/ ICAM-I interactions occur in space and are dependent on activation time but show differences in number, arrangement and fluorescence intensity, depending on time and experimental conditions. LFA-I and ICAM-I adhesion proteins seem to be sensitive to low gravity, without being altered their interaction, therefore reduced lymphocytes activation has to be investigated on downstream molecules of signal transduction pathway. Our results confirm that the space environment on board ISS can provide a platform for basic and applied research and an extraordinary laboratory, where phenomena and biological processes can be studied in the absence of gravity. $T$ lymphocytes are also a good model to fulfill several goals in gravitational biology. In space living organisms, including cells, are affected by two new environmental conditions: microgravity and cosmic radiations. Only an exhaustive perception of the induction/repression profiles of specific genes following exposure of cells to radiations will help to disclose the mechanisms that govern cellular response to this injury. Since ionizing radiations are of fundamental interest for space missions and ISS utilization, the aim of this work was to discriminate between the effects of microgravity and cosmic radiation in $\mathrm{T}$ cells. In order to evaluate the effects of cosmic radiations on the gene expression in human $T$ lymphocytes we exposed these cells to high quote cosmic radiation during two stratospheric balloon transmediterranean flights (BIRBA missions). The gene expression was analyzed by CDNA microarray hybridization technology, using a system of high-density filter-based cDNA microarrays constituted by single membranes containing approximately 4,000 human ORF of known function (Amundsen, 1999). The cells were boarded on the balloon inside two special containers in order to maintain them in controlled conditions of temperature $\left(37 \pm 1^{\circ} \mathrm{C}\right)$ and pressure $(1 \pm 0.05 \mathrm{bar})$ during the flight. One of the two containers was partially shielded from cosmic radiation (fast neutrons component) in order to verify an eventual influence of the radiation dose and composition on the biological effects observed. An accurate dosimetry system for gamma rays, thermic neutrons and fast neutron components was present on board.After a 22 hours flight the balloon landed in Spain where the biological samples were recovered and total RNA extracted for subsequent analysis. We analyzed the transcriptional modulations induced on the embarked T lymphocytes, compared with appropriate ground controls. Several genes were found to be induced in the activated T-lymphocytes. In this case the inductions were more evident in the cells from the unshielded container, as compared with other cells (HUVEC) utilized from others researchers in the same mission. The most interesting aspect of these data is that a subset of the genes was induced in both kinds of cells. Most of them were already known to be induced by ionizing radiation or to play a role in the cellular response to genotoxic stress (Zhou and Elledge, 2000).Altogether, activated $T$ cells react to the ionizing stress by activating genes involved in cell cycle check-point (PP2A, NT5 and SUPTH), oxidative stress response (GPXI), heat shock proteins production (HSPBI, HSPDI and HSF2) or by repressing genes involved in antigen recognition (HLA-C and HLA-DBQI) Further flights and independent analytical methods (Northern blot, RT-PCR) will be required to confirm the significance of these results.

Actually, experiments at SCK-CEN, Moll (Belgium), are in progress on the effects of ionizing radiations on human blood cells by exposing cultured T lymphocytes from peripheral blood to the radiation environment previously measured inside the International Space Station (ISS). This ISS simulated radiation environment available at SCK-CEN will be obtained by applying similar ISS dose rates using a mixture of pure gamma (Cs-137) and neutron (Cf-252) sources. In order to evaluate the effects of ionizing radiations on the $T$ lymphocyte activation process, two kinds of experiments will be conducted. A first kind of experiments will mime a stratospheric balloon flight in which T cells will be irradiated for $\mathbf{2 4}$ hours in both nonactivated and activated conditions by addition of Con $A$ and anti-CD28. In the second kind of experiments, a journey inside the ISS will be simulated and T lymphocytes will be exposed to simulated radiations for either $30 \mathrm{~min}$ or $4 \mathrm{~h}$ after above activation. Concomitantly, $\mathrm{T}$ cells will be also exposed to continuous radiations for $48 \mathrm{~h}$ before activation.Assuming that ionizing radiation causes direct and indirect cellular effects, partially by causing damage to the DNA structure or altering the signal transduction pathways that may consequently induce cell cycle arrest and apoptotic cell death, $T$ cells will be analyzed by flow cytometry for various parameters: cell size and granularity, caspase- 3 activity, $\mathrm{Bcl}-2$ regulation, cell counting and cell cycle.

\section{Acknowledgments}

The research was supported by Agenzia Spaziale Italiana, Roma (MoMa grant) and Fondazione Banco di Sardegna (Sassari).

\section{References}

Amundsen S.A., 1999. Fluorescent CDNA microarray hybridization reveals complexity and heterogeneity of cellular genotoxic stress responses. Oncogene 18, 3666-3672.

Cogoli A., Tschopp A., Fuchs-Bislin P., 1984. Cell sensitivity in gravity. Science 225: 228-230.

Cooper D., Pellis N.R., 1998. Suppressed PHA activation of T lymphocytes in simulated microgravity is restored by direct activation of protein kinase C. J. Leukoc. Biol. 63: 550-562.

Cubano L.A., Lewis M.L. 200I. Effect of vibrational stress and spaceflight on regulation of heat shock proteins hsp70 and hsp27 in human lymphocytes (Jurkat). J. Leukoc. Biol. 69: 755761.

Maccarrone M., Battista N., Meloni M.A., Bari M., Galleri G., Pippia P., Cogoli A., Finazzi-Agrò A., 2003. Creating conditions similar to those that occur during exposure of cells to microgravity induces apoptosis in human lymphocytes by 5-lipoxygenasemediated mitochondrial uncoupling and cytocrome $c$ release. J. Leukoc. Biol. 73: 472-48I. 
Meloni M.A., Galleri G., Pippia P., Cogoli-Greuter M., 2006. Cytoskeleton changes and impaired motility of monocytes at modelled low gravity. Protoplasma 229: 243-249.

Sciola L., Cogoli-Greuter M., Cogoli A., Spano A., Pippia P., 1999. Influence of microgravity on mitogen binding and cytoskeleton in Jurkat cells. Adv. Space Res. 24: 80I-805.

Walther I., Pippia P., Meloni M.A., Turrini F., Mannu F., Cogoli A.,
1998. Simulated microgravity inhibits the genetic expression of interleukin- 2 and its receptor in mitogen-activated $T$ lymphocytes. FEBS Lett. 436: II5-118.

Wang E. 1999. Age-dependent atrophy and microgravity travel: what do they have in common? FASEB J. I3: SI67-SI74.

Zhou B.S., Elledge S.J., 2000.The DNA damage response: putting checkpoints in perspective. Nature 408:433-439. 